\title{
NECESSARY AND SUFFICIENT CONDITIONS FOR CARL- SON'S THEOREM ON ENTIRE FUNCTIONS
}

\author{
BY \\ L. A. RUBEL
}

1. Introduction. In this paper we investigate the conditions under which which Carlson's theorem on entire functions applies to a set of positive integers. Our principal result is the following theorem.

Theorem 1. Given a set $A$ of positive integers, in order that each entire function $f(z)$ satisfying

$$
\begin{aligned}
f(z) & =O(1) \exp (\tau|z|) \text { for some } \tau<\infty, \\
f(i y) & =O(1) \exp (c|y|) \text { for some } c<\pi, \\
f(n) & =0 \text { for each } n \text { in } A
\end{aligned}
$$

vanish identically, it is necessary and sufficient that

$$
\Delta(A)=1 \text {. }
$$

Here $\Delta(A)$ is the upper density of $A$, defined by $\Delta(A)=\lim \sup _{t \rightarrow \infty} A(t) / t$, where $A(t)$, the counting function of $A$, is defined as the number of integers $n$ in $A$ for which $n \leqq t$. $\delta(A)$, the lower density of $A$, is defined by $\delta(A)$ $=\lim \inf _{t \rightarrow \infty} A(t) / t$. The following optimal gap theorem is a consequence of Theorem 1.

THEOREM 2. In order that there exist a nonconstant function $g(z)$ whose region of regularity includes the full negative real axis, $-\infty \leqq z \leqq 0$, with a power series expansion of the form

$$
g(z)=\sum^{*} a_{n} z^{n},
$$

it is necessary and sufficient that $\delta(A)>0$.

A star on a $\sum$ (or a $\Pi$ ) will be used throughout this paper to indicate that the index of the sum (or the product) is restricted to lie in $A$.

Theorem 1 is an optimal extension of a celebrated theorem of Carlson which states that the only entire function $f(z)$ satisfying (1.1) and (1.2) and such that $f(n)=0$ for each positive integer $n$ is the null function $f(z) \equiv 0$. The necessity of condition (1.4) has been known for some time, but it has long been suspected that (1.4) is not sufficient. Theorem 1 is analogous to a theorem of Fuchs $\left[1\right.$, p. 157] $\left.{ }^{1}\right)$ which concerns functions $f(z)$ regular only in

Received by the editors December 12, 1955.

(1) Numbers in square brackets refer to the bibliography at the end of this paper. 
the half-plane $R(z) \geqq 0$ under a less severe restriction on the set $A$ than that it be a set of positive integers. In $\$ \S 7$ and 13 we discuss the problem of strengthening Theorem 1 to include more general sets.

In the light of this more general problem, the role played in Theorem 1 by $\Delta(A)$ will appear artificial. Indeed, the natural upper density for this problem seems to be the logarithmic block density $L(A)$, defined by

$$
L(A)=\inf _{\lambda>1} \limsup _{x \rightarrow \infty}(\log \lambda)^{-1} \sum_{x \leqq n \leqq \lambda x}^{*} 1 / n .
$$

This point of view is made explicit in the conjecture of $\S 7$. Support for the conjecture is offered by Theorems 3 and 5 .

In $\S \S 8-11$ we investigate the ordering of certain upper densities that occur often in entire function theory. In $\$ 12$, we obtain a partial converse to a theorem of Levinson on the growth of certain Weierstrass products. $\$ 13$ describes certain difficulties that arise if one uses the methods that succeeded in proving Theorem 1 in an attempt to prove the conjecture of $\$ 7$.

2. Proof of Theorem 1. We shall first prove the following intermediate theorems, of which Theorem 1 is an immediate consequence.

Theorem 3. Theorem 1 is true if we replace (1.4) $\Delta(A)=1$ by

$$
L(A)=1 .
$$

THEOREM 4. For any set $A$ of positive integers, $\Delta(A)=1$ if and only if $L(A)$ $=1$.

3. Proof of the sufficiency of the condition $L(A)=1$. We shall assime that $L(A)=1$ and prove that each entire function satisfying (1.1), (1.2), and (1.3) vanishes identically. Suppose, on the contrary, that there exists an entire function $f(z)$ satisfying (1.1), (1.2), and (1.3) and that $f(z) \not \equiv 0$. There is no loss of generality in assuming that $f(0)=1$, for if $f(0) \neq 1$ we may consider the function $f(z) / f(0)$ if $f(0) \neq 0$, or $f(z) K_{0} z^{p}$ if $f$ has a zero of order $p$ at the origin and $K_{0}$ is an appropriate constant. Let the zeros of $f(z)$ be denoted by $z_{1}, z_{2}, z_{3}, \cdots$, where $z_{n}=r_{n} e^{i \theta_{n}}$. We put $N(t)$, the counting function of the zeros of $f$, equal to the number of $z_{n}$ for which $r_{n} \leqq t$, counting multiple zeros according to their multiplicity. We put $B=-(1 / 4)\left(f^{\prime}(0)+\overline{f^{\prime}(0)}\right)$ and choose a positive number $R$. Then the exact form of Carleman's theorem $[1$, p. 2] states

$$
\begin{aligned}
\sum_{r_{n} \leqq R}\left(1 / r_{n}-r_{n} / R^{2}\right) \cos \theta_{n}= & B+\frac{1}{\pi R} \int_{-\pi / 2}^{\pi / 2} \log \left|f\left(R e^{i \theta}\right)\right| \cos \theta d \theta \\
& +\frac{1}{2 \pi} \int_{0}^{R}\left(1 / y^{2}-1 / R^{2}\right) \log |f(i y) f(-i y)| d y .
\end{aligned}
$$

If, in the above formula, we first put $R=t$ and then $R=\lambda t$, subtract the first from the second, and then discard certain terms, we arrive at 


$$
\begin{aligned}
\sum_{t<n \leq \lambda t}^{*} \frac{1}{n} \leqq & \frac{1}{(\lambda t)^{2}} \sum_{r_{n} \leq \lambda t} r_{n}+\frac{1}{\pi \lambda t} \int_{-\pi / 2}^{\pi / 2} \log \left|f\left(\lambda t e^{i \theta}\right)\right| \cos \theta d \theta \\
& -\frac{1}{\pi t} \int_{-\pi / 2}^{\pi / 2} \log \left|f\left(t e^{i \theta}\right)\right| \cos \theta d \theta \\
& +\frac{1}{2 \pi} \int_{t}^{\lambda t}\left(1 / y^{2}-1 /(\lambda t)^{2}\right) \log |f(i y) f(-i y)| d y \\
& +\frac{1}{2 \pi} \int_{0}^{t}\left(1 / t^{2}-1 /(\lambda t)^{2}\right) \log |f(i y) f(-i y)| d y .
\end{aligned}
$$

All the terms on the right side of (3.1) may be estimated by known techniques $[1$, pp. 16, 31]. The estimates and brief sketches of their derivations are given below. The letter $K$ is used to denote constants which are independent of $\lambda$ and $t$ provided that $t \geqq \alpha$ for some fixed $\delta>0$, say $\delta=1$.

\section{Estimate 1.}

$$
\frac{1}{(\lambda t)^{2}} \sum_{r_{n} \leqq \lambda t} r_{n} \leqq K .
$$

Clearly, $(\lambda t)^{-2} \sum_{r_{n} \leqq \lambda t} r_{n} \leqq N(\lambda t) / \lambda t$. By Jensen's theorem [1, p. 2],

$$
\int_{0}^{e R}(N(s) / s) d s=\frac{1}{2 \pi} \int_{-\pi}^{\pi} \log \left|f\left(e R e^{i \theta}\right)\right| d \theta .
$$

Applying (1.1) to the last integral, we get

$$
\int_{0}^{e R}(N(s) / s) d s \leqq K+e R \tau .
$$

But

$$
\int_{0}^{e R}(N(s) / s) d s \geqq \int_{R}^{e R}(N(s) / s) d s \geqq N(R) \int_{R}^{e R}(1 / s) d s=N(R) .
$$

Putting $R=\lambda t$ and combining (3.3) and (3.4), we have $N(\lambda t) / \lambda t \leqq K / \lambda t+e \tau$ $\leqq K$, and (3.2) follows.

Estimate 2.

$$
\frac{1}{\pi \lambda t} \int_{-\pi / 2}^{\pi / 2} \log \left|f\left(\lambda t e^{i \theta}\right)\right| \cos \theta d \theta \leqq K .
$$

This follows directly from (1.1).

\section{Estimate 3.}

$$
\frac{1}{\pi t} \int_{-\pi / 2}^{\pi / 2} \log \left|f\left(t e^{i \theta}\right)\right| \cos \theta d \theta \geqq-K
$$


By Jensen's theorem,

$$
\int_{-\pi}^{\pi} \log \left|f\left(t e^{i \theta}\right)\right| d \theta=\int \log ^{+}+\int \log ^{-} \geqq 0
$$

so that

$$
\begin{aligned}
-\int_{-\pi / 2}^{\pi / 2} \log & \left|f\left(t e^{i \theta}\right)\right| \cos \theta d \theta \\
& \leqq-\int_{-\pi / 2}^{\pi / 2} \log ^{-}\left|f\left(t e^{i \theta}\right)\right| \cos \theta d \theta \leqq-\int_{-\pi / 2}^{\pi / 2} \log ^{-}\left|f\left(t e^{i \theta}\right)\right| d \theta \\
& \leqq-\int_{-\pi}^{\pi} \log ^{-}\left|f\left(t e^{i \theta}\right)\right| d \theta \leqq \int_{-\pi}^{\pi} \log ^{+}\left|f\left(t e^{i \theta}\right)\right| d \theta \leqq K t
\end{aligned}
$$

by (1.1).

Estimate 4.

$$
\frac{1}{2 \pi} \int_{t}^{\lambda t}\left(1 / y^{2}-1 /(\lambda t)^{2}\right) \log |f(i y) f(-i y)| d y \leqq(c / \pi) \log \lambda+K
$$

Estimate 5 .

$$
\frac{1}{2 \pi} \int_{0}^{t}\left(1 / t^{2}-1 /(\lambda t)^{2}\right) \log |f(i y) f(-i y)| d y \leqq K .
$$

Estimates 4 and 5 are direct consequences of (1.2).

We now apply (3.2), (3.5), (3.6), (3.7), and (3.8) to the inequality (3.1) to get

$$
\sum_{t<n \leqq \lambda t}^{*} 1 / n \leqq(c / \pi) \log \lambda+K .
$$

Since $K$ is independent of $t$ for large $t$, it follows that

$$
\limsup _{t \rightarrow \infty} \sum_{t \leqq n \leqq \lambda t}^{*} 1 / n \leqq(c / \pi) \log \lambda+K .
$$

Since $K$ is independent of $\lambda$,

$$
L(A)=\inf _{\lambda>1}(\log \lambda)^{-1} \limsup _{t \rightarrow \infty} \sum_{\lambda \leqq n \leqq \lambda t}^{*} 1 / n \leqq c / \pi .
$$

But $c<\pi$, so that the sufficiency of (2.1) is now established.

4. Proof of the necessity of the condition $L(A)=1$. We will show that if $L(A)<1$, then the entire function $f_{A}(z)=\Pi^{*}\left(1-z^{2} / n^{2}\right)$ satisfies (1.1), (1.2), and (1.3). It is well known that $f_{A}(z)$ satisfies (1.1), (1.2), and (1.3) with any $c>\pi \Delta(A)$. (See $\S 8$.) Hence, if we could show that $\Delta(A) \leqq L_{1}(A)$, our conclusion would be immediate; but, as a matter of fact, Theorem 6 implies the 
opposite inequality $L(A) \leqq \Delta(A)$. Nevertheless, we shall show that if $L_{1}(A)$ $<1$, then $\Delta(A)<1$. This is the only part of our proof of Theorem 1 that makes full use of the fact that $A$ is a set of positive integers.

Lemma 1. Let $\beta(\lambda)$ be so chosen that

$$
\sum_{t \leqq n<\lambda_{t}}^{*} \frac{1}{n} \leqq \beta(\lambda) \log \lambda
$$

for all sufficiently large $t$. Then

$$
\Delta(A) \leqq \frac{\lambda-\lambda^{1-\beta(\lambda)}}{\lambda-1} .
$$

Furthermore, there exists a set $A$ of positive integers for which we may choose $\lambda$ and $\beta(\lambda)$ so that equality holds in (4.2).

Proof. Suppose that (4.1) is valid for all $t \geqq M$. We may write any such $t$ as $t=\alpha \lambda^{p}$, where $M \leqq \alpha<\lambda M$ and $p$ is a non-negative integer. We put $B_{k}=A\left(\alpha \lambda^{k+1}\right)-A\left(\alpha \lambda^{k}\right), \quad k=0,1, \cdots, \quad p-1, \quad$ so that $A(t)=A\left(\alpha \lambda^{p}\right) \leqq \alpha$ $+\sum_{k=0}^{p-1} B_{k}$. We estimate the $B_{k}$.

$$
\sum_{\alpha \lambda^{k}+1}^{\alpha \lambda^{k+1}} \frac{1}{n} \geqq \sum_{\alpha \lambda^{k+1}-B_{k+1}}^{\alpha \lambda^{k+1}} \frac{1}{n} \geqq \log \frac{\alpha \lambda^{k+1}}{\alpha \lambda^{k+1}-B_{k}+1} .
$$

The first inequality in (4.3) is valid because there are precisely $B_{k}$ terms in each sum, and the $i$ th term in the second sum does not exceed the $i$ th term in the first sum. The second inequality in (4.3) is elementary. The inequalities (4.3) and (4.1) combine to give us $B_{k} \leqq 1+\alpha \lambda^{k+1}\left(1-\lambda^{-\beta(\lambda)}\right)$. Hence, $A(t)$ $=A\left(\alpha \lambda^{p}\right) \leqq A(\alpha)+p+\alpha\left(1-\lambda^{-\beta(\lambda)}\right)\left(\lambda^{p}-1\right)(\lambda-1)^{-1} \lambda$, so that $A(t) / t \leqq(\lambda$ $\left.-\lambda^{1-\beta(\lambda)}\right)(\lambda-1)^{-1}+o(1)$ as $t \rightarrow \infty$, which is what we were required to prove.

A set for which equality holds in (4.2) is the set $A=\langle 1,4,5,6,7,16$, $17, \cdots, 31,64, \cdots>$ of all integers $n$ which satisfy, for some non-negative integer $q$, the inequality $4^{q} \leqq n<2 \cdot 4^{q}$. Direct calculation shows that $\Delta(A)$ $=2 / 3$. On the other hand, if we choose $\lambda=4$, then $(\log 4)^{-1} \sum_{n=t}^{4 t} * 1 / n \leqq 1 / 2$ for all $t$, so that $(4.1)$ is true with $\beta(4)=1 / 2$. But $(\lambda-1)^{-1}\left(\lambda-\lambda^{1-\beta(\lambda)}\right)$ $=3^{-1}\left(4-4^{1 / 2}\right)=2 / 3=\Delta(A)$.

Lemma 2. If $L(A)<1$, then $\Delta(A)<1$.

Proof. If $L(A)<1$, and we choose $\beta$ so that $L(A)<\beta<1$, we may then choose $\lambda>1$ so that (4.1) is satisfied, and we may conclude from Lemma 1 that $\Delta(A) \leqq(\lambda-1)^{-1}\left(\lambda-\lambda^{1-\beta}\right)<1$. This completes the proof of Theorem 3 .

5. Proof of Theorem 4. We can now complete the proofs of Theorems 1 and 4 by proving Theorem 4 . First, suppose that $\Delta(A)=1$. Then Lemma 2 implies that $L(A)=1$. Suppose, next, that $L(A)=1$. Then each entire function $f(z)$ satisfying (1.1), (1.2), and (1.3) must vanish identically. But if 
$\Delta(A)<1$, then the entire function $f_{A}(z)$ defined in the beginning of $\$ 4$ satisfies (1.1), (1.2), (1.3), and the condition $f_{A}(0)=1$. Hence $\Delta(A)=1$.

6. Proof of Theorem 2. Theorem 2 is an immediate consequence of Theorem 1 and a theorem of Buck [2, pp. 542, 543] which implies the following statement.

BuCK's THEOREM. Given a sequence $\left\{a_{n}\right\}, n=0,1,2, \cdots$, of complex numbers, in order that there exist an entire function $f(z)$ satisfying (1.1) and (1.2) with $f(n)=a_{n}$ for $n=0,1,2, \cdots$, it is necessary and sufficient that the function $g(z)=\sum_{n=0}^{\infty} a_{n} z^{n}$ be regular in a region including the full negative real axis $-\infty \leqq z \leqq 0$.

Thus, there exists a function $g(z)$ satisfying the conditions in Theorem 2 if and only if there exists an entire function $f(z)$ satisfying (1.1) and (1.2) which vanishes over the set $\bar{A}$ of all positive integers $n$ not in $A$. By Theorem 1 , then, such a function $g(z)$ exists if and only if $\Delta(\bar{A})<1$. But $\delta(A)=1-\Delta(\bar{A})$ and we are done.

7. More general sets. In this section we propose the following conjecture. ing

$$
\lambda_{n+1}-\lambda_{n} \geqq \gamma>0
$$$$
\text { for } n=1,2,3, \cdots \text {. }
$$

In order that each entire function $f(z)$ satisfying

$$
\begin{aligned}
f(z) & =O(1) \exp (\tau|z|) \\
f(i y) & =O(1) \exp (c|y|) \\
f\left(\lambda_{n}\right) & =0
\end{aligned}
$$

for some $\tau<\infty$, for some $c<\mathfrak{e}$, for $n=1,2,3, \cdots$

vanish identically, it is necessary and sufficient that

$$
L(\Lambda) \geqq \mathfrak{e} / \pi \text {. }
$$

Here, $L(\Lambda)$ is the logarithmic block density of $\Lambda$ defined by

$$
L(\Lambda)=\inf _{\lambda>1} \limsup _{t \rightarrow \infty}(\log \lambda)^{-1} \sum_{\lambda \leqq \lambda_{n} \leqq \lambda t} 1 / \lambda_{n} .
$$

An examination of the proof in $\S 3$ shows that we have actually proved the sufficiency of (7.5).

THEOREM 5. Let $\Lambda$ satisfy (7.1), and let (7.5) be satisfied. Then each entire function $f(z)$ satisfying (7.2), (7.3) and (7.4) must vanish identically.

Before describing (see \$13) certain unusual difficulties that would have to be met before the necessity of (7.5) could be established, it will be useful to discuss some familiar upper densities.

8. Upper densities. In all that follows, $\Lambda$ will denote an arbitrary set of positive real numbers $\lambda_{n}, n=1,2,3, \cdots$, satisfying the separation condition 
(7.1). Even this restriction is not essential to what follows except that we thereby avoid certain notational and terminological difficulties. We denote by $\Lambda(t)$ the number of $\lambda_{n}$ which do not exceed $t$, and now define all the upper densities we shall refer to. When the set under discussion is known to be a set of positive integers, it will be denoted by $A$, in keeping with our previous notation.

Upper density:

$$
\Delta(\Lambda)=\limsup _{t \rightarrow \infty} \Lambda(t) / t
$$

Upper mean density:

$$
\Delta_{M}(\Lambda)=\limsup _{t \rightarrow \infty} t^{-1} \int_{0}^{t}(\Lambda(x) / x) d x .
$$

Upper Abel density:

$$
\Delta a(\Lambda)=\limsup _{y \rightarrow \infty} y^{-1} \int_{0}^{\infty} e^{-t} d \Lambda(t y) .
$$

Upper Poisson density:

$$
\Delta_{P}(\Lambda)=\pi^{-1} \limsup _{\nu \rightarrow \infty} y^{-1} \int_{0}^{\infty} \log \left(1+1 / t^{2}\right) d \Lambda(t y) .
$$

The corresponding lower densities are defined with lim inf instead of lim sup and are denoted by $\delta$ with an appropriate subscript. If $\delta(\Lambda)=\Delta(\Lambda)$ we say that the density $D(\Lambda)$ exists and put $D(\Lambda)=\delta(\Lambda)=\Delta(\Lambda)$. We use the same convention to define $D_{P}, D_{a}$, and $D_{M}$.

Logarithmic block density:

$$
L(\Lambda)=\lim _{\lambda \rightarrow \infty} \limsup _{t \rightarrow \infty} \lambda^{-1} t^{-1} \int_{1}^{e^{\lambda}} v^{-1} d \Lambda(t v) .
$$

This definition of $L_{1}(\Lambda)$ is equivalent to the definition (7.6), the sum in (7.6) being replaced by a Stieltjes integral, with $\lambda$ replaced by $e^{\lambda}$. The existence of the limit, and its equivalence to the infimum may be shown by familiar arguments $[7$, pp. 559,560$]$.

The entire function $f_{\Delta}(z)=\prod_{n=1}^{\infty}\left(1-z^{2} / \lambda_{n}^{2}\right)$ satisfies the inequality

$$
f_{\Delta}(z)=O(1) \exp (a|x|+c|y|+\epsilon|z|) \quad \text { for each } \epsilon>0
$$

where

$$
a=a(\Lambda)=\limsup _{x \rightarrow \infty} x^{-1} \log \left|f_{\Lambda}(x)\right|
$$

and 


$$
c=c(\Lambda)=\limsup _{y \rightarrow \infty} y^{-1} \log \left|f_{\Lambda}(i y)\right|=\pi \Delta_{P}(\Lambda)
$$

are the smallest numbers $a$ and $c$ for which (8.1) is valid [3, pp. 346-347]. The following inequalities are well known.

$$
\begin{aligned}
\Delta_{P}(\Lambda) & \leqq \Delta_{M}(\Lambda)[3, \text { p. 347] } \\
\Delta_{Q}(\Lambda) & \leqq \Delta_{M}(\Lambda), \\
\Delta_{M}(\Lambda) & \leqq \Delta(\Lambda) .
\end{aligned}
$$

We shall prove the following additional results.

Theorem 6. For any set $\Lambda, L(\Lambda) \leqq \Delta_{P}(\Lambda)$. Furthermore, there exists a set $A$ of positive integers for which $L(A)<\Delta_{P}(A)$.

Theorem 7. For any set $\Lambda, \Delta_{P}(\Lambda) \leqq \Delta_{\mathfrak{Q}}(\Lambda)$. Furthermore there exists a set $A$ of positive integers for which $\Delta_{P}(A)<\Delta_{Q}(A)$.

THEOREM 8. Given a set $A$ of positive integers, if any one of the equalities $L(A)=1, \Delta_{P}(A)=1, \Delta_{\mathfrak{Q}}(A)=1, \Delta_{M}(A)=1, \Delta(A)=1$ holds, then all of them hold.

The second part of Theorem 6 is of particular importance to the general problem of $\S 7$, and we refer the reader to the final section of this paper for a discussion of its significance. Theorem 8 is proved by indirect means. Direct proofs of Theorem 8 insofar as it concerns $\Delta_{M}(A)$ and $\Delta(A)$, and insofar as it concerns $\Delta_{Q}(A)$ and $\Delta_{M}(A)$ have been obtained by Dvoretzky [4], and Agnew (unpublished), respectively.

9. Proof of Theorem 6. Suppose that the first part of the theorem is false. Then there is a set $\Lambda$ for which $\Delta_{P}(\Lambda)<L(\Lambda)$ and we may choose $\epsilon>0$ for which $\Delta_{P}(\Lambda)+\epsilon<L(\Lambda)$. By (8.1), the function $f_{\Lambda}(z)$ satisfies (7.2), (7.3), and (7.4) with $\mathfrak{e}=\pi\left(\Delta_{P}(\Lambda)+\epsilon\right)$. Since $f_{\Lambda}(0)=1, f_{\Lambda}(z)$ certainly does not vanish identically, and we are therefore assured by Theorem 5 that $L(\Lambda)<\mathfrak{e} / \pi$. This is impossible, and the first part of Theorem 6 is established.

An example of a set $A$ of positive integers for which $L(A)<\Delta_{P}(A)$ is the set $A=\langle 1,4,5,6,7,16,17, \cdots, 31,64, \cdots\rangle$ that served as an example for Lemma 2. Our proof will be extremely indirect. We shall need to refer to the following application of Pitt's Tauberian theorem [8, p. 211].

Lemma 3. If $D_{P}(\Lambda)$ exists, then $D(\Lambda)$ exists and $D_{P}(\Lambda)=D(\Lambda)$.

Sketch of proof. After the usual changes of variables and integration by parts, it is seen that we need only establish that $e^{-t} \Lambda\left(e^{t}\right)$ is slowly decreasing and that the Fourier transform of sech $x$ vanishes nowhere. The first part is elementary, and for the second it suffices to say that sech $(\pi / 2)^{1 / 2} x$ is its own Fourier transform.

The function $f_{A}(z)$ corresponding to the set $A$ above obeys the "functional equation" 


$$
f(i y) f(2 i y)^{1 / 2}=(\sin \pi i y) \exp o(|y|) \text { as } y \rightarrow \pm \infty .
$$

We write

$$
\begin{aligned}
\frac{\sin \pi i y}{\pi i y} & =\prod_{n=1}^{\infty}\left(1+y^{2} / n^{2}\right)=\coprod_{n \in A}\left(1+y^{2} / n^{2}\right) \coprod_{n \in A}\left(1+y^{2} / n^{2}\right) \\
& =f_{A}(i y) f_{A}^{*}(i y) .
\end{aligned}
$$

Now

$$
\begin{aligned}
f_{\boldsymbol{A}}^{*}(i y) & =\prod_{k=0}^{\infty}\left\{\prod_{2 \cdot 4^{k} \leqq n<4^{k+1}}\left(1+y^{2} / n^{2}\right)\right\} \\
& =\prod_{k=0}^{\infty}\left\{\prod_{2 \cdot 4^{k} \leqq n<4^{k+1}}\left(1+(2 y)^{2} /(2 n)^{2}\right)\right\} \\
& =\prod_{k=0}^{\infty}\left\{\prod_{4^{k} \leqq m<2 \cdot 4^{k} ; m \equiv 0 \bmod 2}\left(1+(2 y)^{2} / m^{2}\right)\right\} .
\end{aligned}
$$

If we denote by $A_{0}$ the set of all even integers in $A$, and by $A_{1}$ the set of all odd integers in $A$, and put $f_{0}(z)=f_{A_{0}}(z), f_{1}(z)=f_{A_{1}}(z)$, we have $f_{A}(z)=f_{0}(z) f_{1}(z)$. Furthermore, it is easily seen that $f_{0}(i y)=f_{1}(i y) \exp o(|y|)$. Thus, $f_{0}(i y)$ $=f_{A}(i y)^{1 / 2} \exp o(|y|)$. But equation (9.3) states $f_{A}^{*}(i y)=f_{0}(2 i y)$ so that $f_{A}^{*}(i y)=f_{A}(2 i y)^{1 / 2} \exp o(|y|)$ and (9.2) may now be written as

$$
(\pi i y)^{-1} \sin \pi i y=f_{A}(i y) f_{A}^{*}(i y)=f_{A}(i y) f_{A}(2 i y)^{1 / 2} \exp o(|y|),
$$

and (9.1) is therefore valid.

Let us now put $g_{A}(y)=y^{-1} \log f_{A}(i y)$ so that (9.1) becomes $g_{A}(y)+g_{A}(2 y)$ $=\pi+o(1)$. Hence $\pi=\lim \sup _{y \rightarrow \infty} g(y)+\lim \inf _{y \rightarrow \infty} g(y)=\pi \Delta_{P}(A)+\pi \delta_{P}(A)$. Thus $\delta_{P}(A)+\Delta_{P}(A)=1$. But $L(A)=1 / 2$, so that if $\Delta_{P}(A)=L(A)=1 / 2$, it follows that $\left(\delta_{P}(A)=\Delta_{P}(A)=D_{P}(A)=1 / 2\right.$, and it would follow from Lemma 3 that $D(A)=1 / 2$. We have seen, however, that $\Delta(A)=2 / 3 \neq 1 / 2$. Thus, we are forced to the conclusion that $\Delta_{P}(A)>L(A)$.

10. Proof of Theorem 7. Our proof will depend on the fact that $\log \left(1+1 / t^{2}\right)$ is the Laplace transform of a non-negative function: $\log \left(1+1 / t^{2}\right)$ $=\int_{0}^{\infty} e^{-t x} K(x) d x$, where $K(x)=2 x^{-1}(1-\cos x) \geqq 0$. We wish to compare

$$
\Delta_{P}(\Lambda)=\pi^{-1} \limsup _{y \rightarrow \infty} y^{-1} \int_{0}^{\infty} \log \left(1+1 / t^{2}\right) d \Lambda(t y)
$$

with

$$
\Delta Q(\Lambda)=\limsup _{y \rightarrow \infty} y^{-1} \int_{0}^{\infty} e^{-t} d \Lambda(t y) .
$$

We write 


$$
\begin{aligned}
(\pi y)^{-1} \int_{0}^{\infty} \log \left(1+1 / t^{2}\right) & d \Lambda(t y)=(\pi y)^{-1} \int_{0}^{\infty} \int_{0}^{\infty} e^{-t x} K(x) d x d \Lambda(t y) \\
= & (\pi y)^{-1} \int_{0}^{\infty}\left\{\int_{0}^{\infty} e^{-t x} d \Lambda(t y)\right\} K(x) d x \\
& =\pi^{-1} \int_{0}^{\infty}\left\{(y / x)^{-1} \int_{0}^{\infty} e^{-w} d \Lambda(w \cdot y / x)\right\}(K(x) / x) d x .
\end{aligned}
$$

Hence,

$$
\Delta_{P}(\Lambda) \leqq \limsup _{v \rightarrow \infty}\left\{v^{-1} \int_{0}^{\infty} e^{-w} d \Lambda(w v)\right\} \pi^{-1} \int_{0}^{\infty}(K(x) / x) d x=\Delta_{Q}(\Lambda)
$$

since $\int_{0}^{\infty}(K(x) / x) d x=\pi$.

We remark that if we define

$$
\Delta_{k}(\Lambda)=\limsup _{y \rightarrow \infty} y^{-1} \int_{0}^{\infty} k(t) d \Lambda(t y),
$$

where $\int_{0}^{\infty} k(t) d t=1$, then in order that $\Delta_{k}(\Lambda) \leqq \Delta_{Q}(\Lambda)$ for each set $\Lambda$, it is sufficient that $k(x)$ be completely monotonic for $0 \leqq x<\infty$. For by a theorem of Bernstein [8, p. 160] we may write any such $k(x)$ as $\int_{0}^{\infty} e^{-x t} d \mathscr{K}(t)$, where $\mathcal{K}(t)$ is bounded and nondecreasing, and substantially repeat the above proof.

We shall now construct a set $A$ of positive integers for which $\Delta_{P}(A)$ $<\Delta_{Q}(A)$. Let us define $A_{\lambda}(\lambda>1)$ as the set of all positive integers $n$ for which there exists an integer $k$ such that $n_{k} \leqq n \leqq \lambda n_{k}$, where $n_{k}=2^{2^{k}}$. (Direct calculation shows that $\Delta_{M}\left(A_{\lambda}\right)=\Delta_{Q}\left(A_{\lambda}\right)=(\lambda-1) \lambda^{\lambda /(1-\lambda)}$.) We will show that for $\lambda$ sufficiently close to $1, \Delta_{P}\left(A_{\lambda}\right)<\Delta_{Q}\left(A_{\lambda}\right)$. We shall write

$$
\Delta_{P}(A)=\limsup _{y \rightarrow \infty} y^{-1} \int_{0}^{\infty} K_{1}(t / y) d A(t)
$$

and

$$
\Delta Q(A)=\limsup _{y \rightarrow \infty} y^{-1} \int_{0}^{\infty} K_{2}(t / y) d A(t)
$$

where $K_{1}(x)=\pi^{-1} \log \left(1+1 / x^{2}\right)$ and $K_{2}(x)=e^{-x}$. It is easily verified that we have chosen the sequence $\left\{n_{k}\right\}=2^{2^{k}}$ of sufficiently rapid growth that

$$
\begin{aligned}
& y^{-1} \int_{0}^{\infty} K_{1}(t / y) d A(t)=y^{-1} \int_{n_{k}}^{\lambda n_{k}} K_{1}(t / y) d t+o(1) \\
& y^{-1} \int_{0}^{\infty} K_{2}(t / y) d A(t)=y^{-1} \int_{n_{k}}^{\lambda_{n k}} K_{2}(t / y) d t+o(1)
\end{aligned}
$$


where $n_{k}=n_{k}(y)$ begins the block of consecutive integers in $A$ to which $y$ is nearest. If we change variables in (10.1) and (10.2), we see.that

$$
\begin{aligned}
& \Delta_{P}\left(A_{\lambda}\right)=\sup _{-\infty<\phi<\infty} \int_{\phi}^{\phi+l} E_{1}(v) d v, \\
& \Delta Q\left(A_{\lambda}\right)=\sup _{-\infty<\phi<\infty} \int_{\phi}^{\phi+l} E_{2}(v) d v
\end{aligned}
$$

where $l=\log \lambda$ and

$$
\begin{aligned}
& E_{1}(v)=\pi^{-1} e^{v} \log \left(1+e^{-2 v}\right), \\
& E_{2}(v)=e^{v} \cdot e^{-0 v} .
\end{aligned}
$$

An elementary computation shows us that

$$
\sup _{-\infty<v<\infty} E_{1}(v)<\sup _{-\infty<v<\infty} E_{2}(v)
$$

so that if we make $l$ sufficiently small by choosing $\lambda$ sufficiently close to 1 , we have

$$
\inf _{|v-v 0| \leq l / 2} E_{2}(v)>\sup _{-\infty<i<\infty} E_{1}(v),
$$

where $v_{0}$ is so chosen that

$$
E_{2}\left(v_{0}\right)=\sup _{-\infty<v<\infty} E_{2}(v) .
$$

Hence, for such a choice of $\lambda$, we have

$$
\begin{aligned}
\Delta_{P}\left(A_{\lambda}\right) & =\sup _{-\infty<\phi<\infty} \int_{\phi}^{\phi+l} E_{1}(v) d v \leqq l \sup _{-\infty<v<\infty} E_{1}(v) \\
& <l \inf _{|,-, v| \leqq l / 2} E_{2}(v) \leqq \int_{v_{0}-l / 2}^{v_{0}+l / 2} E_{2}(v) d v \\
& \leqq \sup _{-\infty<\phi<\infty} \int_{\phi}^{\phi+l} E_{2}(v) d v=\Delta Q\left(A_{\lambda}\right) .
\end{aligned}
$$

and we are done.

11. Proof of Theorem 8. Let us condense (8.2), (8.3), (8.4), and the first inequalities of Theorems 6 and 7 into a single inequality, valid for all sets $\Lambda$.

$$
L(\Lambda) \leqq \Delta_{P}(\Lambda) \leqq \Delta a(\Lambda) \leqq \Delta_{M}(\Lambda) \leqq \Delta(\Lambda) .
$$

For sets $A$ of positive integers, we may write

$$
L(A) \leqq \Delta_{P}(A) \leqq \Delta_{Q}(A) \leqq \Delta_{M}(A) \leqq \Delta(A) \leqq 1 .
$$


But Theorem 4 tells us that $L(A)=1$ if and only if $\Delta(A)=1$ and the proof is now immediate.

12. Weierstrass products of positive real exponential growth. Levinson [6, p. 92] has shown that if $D(\Lambda)$ exists, then the Weierstrass product $f_{\Lambda}(z)=\prod_{n=1}^{\infty}\left(1-z^{2} / \lambda_{n}^{2}\right)$ has exponential rate of growth zero along the real axis, that is, $a(\Lambda)=\lim \sup _{x \rightarrow \infty} x^{-1} \log |f(x)|=0$. We shall now prove a partial converse of Levinson's result, to the effect that if $a(\Lambda)=0$, then $\Lambda$ may not be so irregular that the Poisson and mean upper densities of $\Lambda$ are different from each other.

THEOREM 9. If $\Delta_{P}(\Lambda)<\Delta_{M}(\Lambda)$ then $a(\Lambda)>0$. Indeed, $a(\Lambda) \geqq \pi\left(\Delta_{M}(\Lambda)\right.$ $\left.-\Delta_{P}(\Lambda)\right)$.

Proof. By Jensen's theorem, observing that the number of zeros of $f_{\mathbf{\Lambda}}(z)$ in $|z| \leqq t$ is $2 \Lambda(t)$, we have

$$
\int_{0}^{r}(2 \Lambda(t) / t) d t=\frac{1}{2 \pi} \int_{-\pi}^{\pi} \log \left|f_{\Lambda}\left(r e^{i \theta}\right)\right| d \theta
$$

But, by (8.1), we have

$$
\log \left|f_{\Lambda}\left(r e^{i \theta}\right)\right| \leqq(a|\cos \theta|+c|\sin \theta|+\epsilon) r+O(1)
$$

where $a=a(\Lambda), c=c(\Lambda)=\pi \Delta_{P}(\Lambda)$, and $\epsilon>0$ is arbitrary. Applying (12.2) to the second integral in (12.1), we have

$$
\frac{2}{r} \int_{0}^{r}(\Lambda(t) / t) d t \leqq 2 \frac{a+c}{\pi}+\epsilon+o(1),
$$

so that

$$
\Delta_{M}(\Lambda) \leqq \frac{a+c}{\pi} .
$$

But $c / \pi=\Delta_{P}(\Lambda)$ and $\Delta_{P}(\Lambda) \leqq \Delta_{M}(\Lambda)$, so that

$$
\frac{c}{\pi}=\Delta_{P}(\Lambda) \leqq \Delta_{M}(\Lambda) \leqq \frac{a+c}{\pi}
$$

and the proof is complete.

The example of Theorem 7 provides us with a set $A$ of positive integers for which $f_{A}(z)$ has a positive rate of exponential growth along the real axis.

13. Remarks on the conjecture. In the proof of Theorem 1, we saw that if there exists any nonidentically vanishing entire function $f(z)$ which satisfies the growth conditions (1.1) and (1.2) and which vanishes over the given set $A$ of positive integers, then the Wierstrass product $f_{A}(z)=\Pi^{*}\left(1-z^{2} / n^{2}\right)$ is such a function. We would like to point out that any attempt to prove the necessity of (7.5) in the conjecture of $\S 7$ by using $f_{\Lambda}(z)=\prod_{n=1}^{\infty}\left(1-z^{2} / \lambda_{n}^{2}\right)$ as 
$f_{A}(z)$ was used in the proof of Theorem 1 must necessarily fail. To see this, we need only take for our set $\Lambda$ the set $A$ that was our example for Theorem 6 . Since $L(\Lambda)<\Delta_{P}(\Lambda)$, we may choose $\mathcal{e}$ so that $L(\Lambda)<\mathfrak{e} / \pi<\Delta_{P}(\Lambda)$. Thus, if we believe in the conjecture, we believe that there exists some entire function $f(z)$ which satisfies the growth conditions (7.2) and (7.3), which vanishes over the set $\Lambda$, and which is not identically zero. But $f_{\Lambda}(z)$ cannot be such a function! This is because $\pi \Delta_{P}(\Lambda)>\mathbb{e}$ so that $f_{\Lambda}(z)$ violates (7.3).

\section{BibLIOGRAPHY}

1. R. P. Boas, Jr., Entire functions, New York, Academic Press, 1954.

2. R. C. Buck, $A$ class of entire functions, Duke Math. J. vol. 13 (1946) pp. 541-559.

3. - An extension of Carlson's theorem, Duke Math. J. vol. 13 (1946) pp. 345-349.

4. A. Dvoretzky, Sur les suites d'exposants d densité supérieure finie, C. R. Acad. Sci. Paris vol. 225 (1947) pp. 481-483.

5. G. H. Hardy, Divergent series, Oxford, Clarendon Press, 1949.

6. N. Levinson, Gap and density theorems, New York, American Mathematical Society, 1940.

7. G. Polya, Untersuchungen über Lücken und Singularitäten von Potenzreihen, Math. Zeit. vol. 29 (1929) pp. 549-640.

8. D. V. Widder, The Laplace transform, Princeton, Princeton University Press, 1946.

CORNELl University, ITHACA, N.Y. 